\title{
Development of Fashion Product Evaluation (FPE) Framework
}

Armine Ghalachyan and Elena Karpova, Iowa State University, USA

Keywords: Evaluative criteria, fashion product evaluation, framework

Background. To develop successful products, it is important to understand how consumers perceive and evaluate apparel and what criteria they use in the process. Product attributes or cues consumers use to examine apparel and make purchase decisions are known as evaluative criteria (Abraham-Muralli \& Littrell, 1995; Eckman et. al, 1990; Jeong \& Lee, 2014; Swinker \& Hines, 2006). Examination of various apparel evaluative criteria and existing classifications reveal an array of overlapping and unclear categories and dimensions of product attributes. The purpose of this research was to develop an integrated and comprehensive classification system that can be effectively used by researchers and industry professionals for development and evaluation of fashion products.

A common approach for classifying apparel evaluative criteria is to group them into: (a) intrinsic criteria, or attributes that are inherent, physical part of product (e.g., fiber content, color); and (b) extrinsic criteria, which are not a physical part of product (e.g., price, brand). Extrinsic criteria are also referred to as retailer- or manufacturer-defined attributes (Forney, Park \& Brandon, 2005). However, extrinsic criteria category has also included attributes like 'approval of others' or 'coordination with wardrobe' (Eckman et al., 1990), which are consumer-ascribed attributes. Similarly, under the intrinsic category, some researchers have included only product structural and physical attributes, such as fiber content and construction (e.g., Swinker \& Hines, 2006), whereas others included product performance attributes such as comfort and fit (e.g., Jeong \& Lee, 2014).

More complex classification approaches have also been proposed in an attempt to organize the multitude of apparel evaluative criteria (Abraham-Murali \& Littrell, 1995; Eckman et al., 1990; Fiore \& Damhorst, 1992; Fiore \& Ogle, 2000). Existing frameworks used different classification logic and rules, which often were not justified. A need for a comprehensive and systematic classification approach that integrates and logically organizes various apparel evaluative criteria and clarifies the descriptions and labeling of both criteria and categories is apparent.

FPE Framework. Based on a systematic review and analysis of extant research on apparel evaluative criteria, a new framework for Fashion Product Evaluation (FPE) was developed (Figure). FPE framework integrates all apparel evaluative criteria

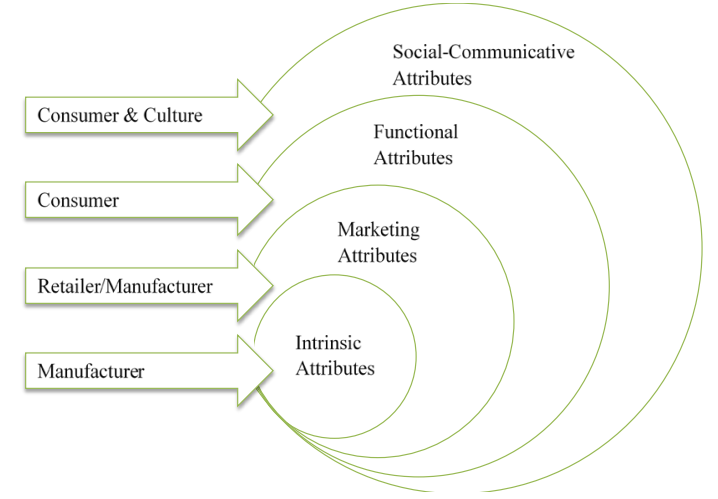

Figure. Dimensions and interactions in Fashion Product Evaluation (FPE) framework.

Published under a Creative Commons Attribution License (https://creativecommons.org/licenses/by/4.0/), which permits unrestricted use, distribution, and reproduction in any medium, provided the original work is properly cited.

ITAA Proceedings, \#76 - https://itaaonline.org 
into four clearly defined dimensions. Following procedural steps for theory development (Hunt, 2002), law-like generalizations and propositions were formulated to define each dimension and guide the classification of evaluative criteria.

First, evaluative criteria identified from extant research through content analysis were recorded, evaluated and constantly compared to other criteria, helping to clarify them and eliminate redundancies. For example, situational appropriateness, appropriateness, and appropriateness to lifestyle attributes were combined under the suitability attribute. Next, the final list of the distinct evaluative criteria were grouped into two broad categories: tangible and intangible criteria. Finally, tangible and intangible criteria were categorized into four mutually exclusive dimensions (Table).

All tangible criteria constitue one dimension: intrinsic attributes. These are inherent, tangible parts of a product (e.g., color, shape), and changing any of them would physically alter the product. These attributes relate to product appearance, composition, and structure and are observable or perceivable through senses. Intrinsic attributes are defined by producers and retailers during product development and manufacturing stages.

Intangible criteria were classified into three distinct dimensions: marketing attributes, functional attributes, and social-communicative attributes (Table). Marketing attributes are defined by retailers or manufacturers and aid in promoting and selling products: price, brand, country of origin, service, etc. Functional attributes are instrumental outcomes of consumer-product interaction during product evaluation and use: durability, quality, comfort, protection, etc. Functional attributes are enabled by intrinsic criteria. For example, a thicker fabric of a certain fiber content (intrinsic attributes) can enable greater durability and protection from harsh weather.

Social-communicative attributes relate to product symbolic and communicative aspects. They allow consumers to express or communicate their identities, values, beliefs in the process of social interactions. This dimension includes attributes such as fashionability, pleasing to others, suitability, and uniqueness. Social-communicative attributes are enabled through complex interactions of tangible and intangible product attributes and mediated by culture. Consumers go through complex mental and intuitive processes to make inferences about social-communicative product attributes, synthesizing information about tangible and intangible product attributes and interpreting them through the lenses of societal and cultural norms.

Page 2 of 3

Published under a Creative Commons Attribution License (https://creativecommons.org/licenses/by/4.0/), which permits unrestricted use, distribution, and reproduction in any medium, provided the original work is properly cited.

ITAA Proceedings, \#76 - https: //itaaonline.org 
Conclusions and Implications. FPE framework is the first to integrate apparel evaluative criteria into a comprehensive classification system consisting of four mutually exclusive dimensions. Each dimension is enabled by different interactions between product, producer, consumer, and society/culture. The framework was tested by using it to examine consumer perceptions of an innovative fashion product, utilizing focus groups. The dimensions of the framework were successfully used to guide the focus group discussions as well as to analyze and categorize the findings.

The framework can be useful for examining consumer perceptions, evaluations, and decisionmaking processes related to fashion products such as clothing, footwear, and accessories. It can help to identify gaps between consumer needs and existing products, resulting in new or improved products that address consumer expectations. The framework also helps to clarify and simplify the use of evaluative criteria by researchers and practitioners.

\section{References}

Abraham-Murali, L., \& Littrell, M. A. (1995). Consumers' conceptualization of apparel attributes. Clothing and Textiles Research Journal, 13, 65-74.

Eckman, M., Damhorst, M. L. \& Kadolph, S. J. (1990). Toward a model of the in-store purchase decision process: Consumer use of criteria for evaluating women's apparel. Clothing and Textiles Research journal, 8(2), 13-22.

Fiore, A. M. \& Damhorst, M. L. (1992). Intrinsic cues as predictors of perceived quality of apparel. Journal of Consumer Satisfaction, Dissatisfaction and Complaining Behavior, 5 , 168-178.

Fiore, A. M., \& Ogle, J. P. (2000). Facilitating students' integration of textiles and apparel subject matter. Part one: Dimensions of a model and taxonomy. Clothing and Textiles Research Journal, 18(1), 31-45.

Forney, J. C., Park, E. J., \& Brandon, L. (2005). Effects of evaluative criteria on fashion brand extension. Journal of Fashion Marketing and Management, 9(2), 156-165.

Hunt, S. (2002). Foundations of marketing theory: Towards a general theory of marketing. Armonk, NY: M.E. Sharpe.

Jeong, S. W., \& Lee, K.-H. (2014). Impact of evaluative criteria on satisfaction and dissatisfaction: Identifying the role of knitwear involvement. Fashion and Textiles, 1(9).

May-Plumlee, T. \& Little, T. J. (2006). Proactive product development integrating consumer requirements. International Journal of Clothing Science and Technology, 18(1), 53-66.

Swinker, M. E. \& Hines, J. D. (2006). Understanding consumers' perception of clothing quality: A multidimensional approach. International Journal of Consumer Studies, 30, 218-223.

Page 3 of 3

(c) 2019 The author(s). Published under a Creative Commons Attribution License (https://creativecommons.org/licenses/by/4.0/), which permits unrestricted use, distribution, and reproduction in any medium, provided the original work is properly cited.

ITAA Proceedings, \#76 - https://itaaonline.org 